\title{
O Intervalo de Tempo para uma Máxima Previsiblidade da Precipitação sobre o Semiarido Brasileiro
}

\author{
Samuellson Lopes Cabral ${ }^{1}$, José Nilson B. Campos ${ }^{2}$, Cleiton da Silva Silveira ${ }^{3}$, \\ José Marcelo Rodrigues Pereira ${ }^{4}$ \\ ${ }^{1}$ Centro Nacional de Monitoramento e Alertas de Desastres Naturais, \\ São José dos Campos, SP, Brasil. \\ ${ }^{2}$ Departamento de Engenharia Hidráulica e Ambiental, Universidade Federal do Ceará, \\ Fortaleza, CE, Brasil. \\ ${ }^{3}$ Instituto de Engenharias e Desenvolvimento Sustentável, \\ Universidade da Integração Internacional da Lusofonia Afro-Brasileira, Redenção, CE, Brasil. \\ ${ }^{4}$ Fundação Cearense de Meteorologia e Recursos Hídricos, Fortaleza, CE, Brasil.
}

Recebido: 26/8/2013 - Aceito: 6/7/2015

\begin{abstract}
Resumo
O artigo apresenta uma avaliação do desempenho na previsão climática no alto vale do rio Jaguaribe, Estado do Ceará. A previsão é executada utilizando as condições de fronteira do modelo de circulação global (ECHAM 4.5) ao modelo regional (RAMS 6.0). O modelo simula as chuvas em escala mensal para agregá-las para a estação chuvosa regional (janeiro a junho). Utilizou-se, o Heidke Skill Score (HSS) como métrica da habilidade de previsão. As simulações foram feitas para a estação úmida abrangendo o período de 1979 a 2010. O HSS foi estimado para intervalos de 1, 2, 10, 15, 21, 30, 45, 60, 120 e 180 dias com o objetivo de encontrar o Intervalo de Tempo de Máxima Previsibilidade (ITEMP). Obteve-se uma curva HSS vs. tempo com máximo no ponto HSS = 0,62 e 45 dias. O valor do HSS obtido, 0,62, mostra que a previsão climática mesmo com suas limitações (dados inicial de umidade do solo e parametrizações utilizadas) pode ser uma importante ferramenta para a gestão de estoques de água de reservatórios do semiárido.
\end{abstract}

Palavras-chave: previsão climática, Heidke Skill Score, operação de reservatório.

\section{The Time Interval for Maximum Predictability of Precipitation over Brazilian Semi Arid}

\begin{abstract}
This paper presents an evaluation of the climate forecast in the upper valley of the river Jaguaribe, State of Ceara-Brazil. The forecast was performed using the global circulation model (ECHAM 4.5) boundary conditions to the regional model (RAMS 6.0). The model simulates the monthly scale rainfall in order to aggregate it in the regional rainy season (January to June). We used the Heidke Skill Score (HSS) as a metric of forecast skill. The simulations were performed for the wet season, covering the period from 1979 to 2010 . The HSS was calculated for intervals of $1,2,10,15,21,30,45,60,120$ and 180 days in order to find the Time Interval of Maximum Predictability (ITEMP). The obtained curve, HSS vs. time, presented a maximum HSS of 0.62 for 45 days. This result indicates that climate prediction even with its limitations (initial data of soil moisture and used parameterization) could be an important tool for the reservoir management in Brasilian Semiarid region.
\end{abstract}

Keywords: climate forecast, Heidke Skill Score, reservoir management.

Autor de correspondência: Samuellson Lopes Cabral, e-mail: samuellson.cabral@cemaden.gov.br. 


\section{Introdução}

Quantificar a distribuição das precipitações sobre a terra é de suma importância para a compreensão da hidrologia de superfície. Essa quantificação pode desempenhar um importante papel na previsão e no monitoramento de fenômenos hidrometeorológicos. Entretanto, muitas das áreas propensas a esses fenômenos, sofrem com falta de dados pluviométricos confiáveis e sem falhas.

As regiões semiáridas apresentam altas variabilidades espaciais e temporais das precipitações. Especificamente, o semiárido brasileiro tem sofrido extremos do clima, como as secas e as cheias. A previsão climática constitui-se em uma ferramenta em desenvolvimento para atenuar os impactos negativos causados por esses eventos extremos, para isso utilizam-se tais previsões para antecipar os fenômenos e organizar ações mitigadoras.

Segundo Paz e Collischonn (2011), um dos principais produtos de tais modelos são os campos de chuva calculados para uma grade de pontos ao nível da superfície. São realizadas simulações de chuva com horizontes de alguns dias até meses, de grande utilidade em áreas como previsão de safras agrícolas, previsão e controle de enchentes e planejamento da operação de reservatórios, entre outras.

A previsão de precipitação para períodos sazonais, atualmente apresenta boa confiabilidade em várias áreas do globo, incluindo o Nordeste do Brasil, e em particular sua região semiárida. Este aumento da destreza dos modelos deve-se entre outros fatores a um melhor entendimento das conexões dos oceanos com a atmosfera (GODDARD et al., 2001).

Os atuais modelos de circulação geral da atmosfera forçados por temperaturas da superfície do mar (TSMs) tem a capacidade de capturar a circulação de grande escala da atmosfera sobre o nordeste da América do Sul, entretanto, e são capazes de prever ou simular características da atmosfera ou da precipitação em uma escala mais local, devido a sua resolução espacial (NOBRE et al., 2001).

De acordo com Oliveira (2006), a incompatibilidade de escala temporal e espacial da informação meteorológica restringia seu uso em modelos hidrológicos, tendo uma alternativa nos anos recentes o uso de downscaling dinâmico.

O downscaling usa modelos regionais aninhados a modelos dinâmicos de circulação geral da atmosfera. Os modelos regionais incorporam características locais como topografia, vegetação, solo etc., não contidas nos modelos globais. Dessa forma, os modelos globais respondem mais à influência da circulação de grande escala, enquanto os modelos regionais tentam reproduzir as forçantes dos efeitos locais, mas também são influenciados pelas forçantes do modelo global (Costa e Menezes Neto, 2009). Vários estudos acerca do uso desta técnica downscaling sugerem um melhor desempenho na simulação e previsão de distribuição de precipitação localizada (NOBRE et al., 2001; SUN et al., 2005).
A previsão de alta resolução de sistemas atmosféricos é caracterizada por uma crescente complexidade, que torna os resultados da validação e interpretação dos modelos cada vez mais exigentes, devido principalmente à dificuldade dos processos na superficie. Nessas superficies a densidade limitada de estações meteorologicas não permite uma avaliação precisa das previsões e simulações de alta resolução. Weaver et al., 2002, em seu estudo mostrou que a especificação correta da superfície na modelagem é extremamente importante para os resultados, assim como as configurações de parametrização do modelo atmosférico.

No Nordeste do Brasil, Sun et al. (2005) analisaram simulações entre janeiro-junho utilizando um modelo regional aninhado ao ECHAM 4.5 (European-Hamburg Model version 4.5) e utilizando a temperatura da superfície do mar (TSM) observada, para um período de 30 anos (1971-2000). Os autores destacaram que o modelo resolve bem tanto o padrão espacial como a variabilidade interanual da chuva sobre o Nordeste do Brasil. As mesmas simulações foram avaliadas por Alves et al., 2005, os quais mostraram que para a quadra chuvosa (fevereiro a maio) o ECHAM 4.5 subestimou os valores da precipitação, enquanto o modelo regional corrigiu as previsões do ECHAM 4.5 na costa leste do Nordeste, prevendo valores de chuva mais próximos do observado.

Consequentemente ferramentas avançadas para a validação dos modelos atmosféricos são necessárias e de grande interesse para os meteorologistas e hidrólogos (JASPER e KAUFMANN, 2003).

Para a escala de bacia hidrográfica, Alves et al. (2008) apresentam resultados do potencial do uso da simulação downscaling dinâmico em estimar a variabilidade intrassazonal das chuvas na bacia hidrográfica do açude Castanhão no Ceará. Estudos nos Estados Unidos e Inglaterra têm mostrado bons resultados do downscaling dinâmico na escala de bacia hidrográfica (MILLER et al., 1999).

Entretanto, faz-se necessária a análise das estimativas de chuvas desses modelos climáticos, os quais precisam ser avaliados quanto à destreza em corretamente reproduzir a chuva observada em nível de bacia hidrográfica, com a utilização de índices de pontuação de habilidade, conhecidos também como Skill Score.

Muitos dos índices têm sido usados para avaliar a precisão das simulações/previsões oriundas dos modelos de tempo e clima, entre eles o Heidke Skill Score (HSS) utilizado por Alves et al. (2012) na previsão de vazões na bacia hidrográfica do alto Jaguaribe, Ceará. O HSS mede a destreza da simulação e da previsão para antever a precipitação correta em relação a um padrão particular.

Nesse sentido, este trabalho tem como objetivo determinar o intervalo de tempo de máxima previsibilidade (ITEMP), do modelo regional RAMS 6.0 (Regional Atmospheric Modeling System), por meio do HSS, para as simulações de precipitação em um horizonte de até 180 dias 
(seis meses). Comparam-se as chuvas geradas com séries de precipitações médias observadas, estimadas pelo método de Thiessen.

\section{Dados e Métodos}

\subsection{Bacia do Alto Jaguaribe}

A sub-bacia do alto Jaguaribe (Fig. 1) localiza-se na porção sudoeste do Estado do Ceará. Das cinco sub-bacias que compõem a bacia do Jaguaribe (Alto, Médio e Baixo Jaguaribe, Banabuiú e Salgado) é a que possui maior área. $\mathrm{O}$ rio Jaguaribe tem nascente no município de Tauá e percorre cerca de $633 \mathrm{~km}$ até sua foz no município de Fortim. Ao longo de seu curso existem três grandes reservatórios, o qual percorre uma extensão de aproximadamente $325 \mathrm{~km}$ até alcançar o açude Orós, principal reservatório desta sub-bacia. A superfície de drenagem da bacia é de $24.538 \mathrm{~km}^{2}$, equivalente a $16 \%$ do território cearense.

Climatologicamente, a parte alta da bacia, setor sul do Estado, recebe chuvas no mês de janeiro, principalmente provenientes da influência da incursão de frentes frias que se localizam no setor centro-norte do Nordeste. A partir de fevereiro as chuvas são provenientes da Zona de Convergência Intertropical (ZCIT), com maior intensidade nos meses de março e abril. A bacia passa por um longo período de estiagem, de julho a novembro, com baixíssima pluviosidade e rios totalmente secos. As altitudes nesta região variam entre $250 \mathrm{~m}$ e $400 \mathrm{~m}$ e o clima é semiárido quente, com precipitações médias anuais entre $500 \mathrm{~mm}$ e $700 \mathrm{~mm}$, com acentuada irregularidade no tempo e no espaço. As vazões médias anuais no rio Jaguaribe são de $24,45 \mathrm{~m}^{3} / \mathrm{s}$, porém o período de vazão nula, ou quase nula, estende-se de julho a dezembro (Fig. 2).

Descrevem-se a seguir os dados e o procedimento de comparação entre pares de campos de chuvas observadas e estimadas referentes a um mesmo instante de tempo. Apresenta-se também a avaliação do desempenho das estimativas.

Os dados e métodos utilizados neste trabalho são apresentados nas seis seguintes subseções: (1) dados de precipitação, (2) cálculo da precipitação média diária pelo método de Thiessen, (3), modelagem dinâmica da precipitação, (4) análise dos membros do RAMS 6.0, (5) a analise da eficiência dos modelos com o índice HSS e (6) a determinação do ITEMP. A Fig. 3 mostra a representação esquemática da metodologia aplicada na bacia hidrográfica do alto Jaguaribe.

\subsection{Dados das precipitações}

Os dados pluviométricos utilizados nesse artigo são disponibilizados pela Fundação Cearense de Meteorologia e Recursos Hídricos (FUNCEME), em www.funceme.br. No presente estudo foram utilizadas séries históricas de 65 estações pluviométricas inseridas na área da bacia do Alto Jaguaribe. Os dados englobam o período de $1^{\circ}$ de janeiro de 1979 a 31 de dezembro de 2010, totalizando 32 anos de dados, caracterizando claramente a climatologia da bacia hidrográfica em todo período.

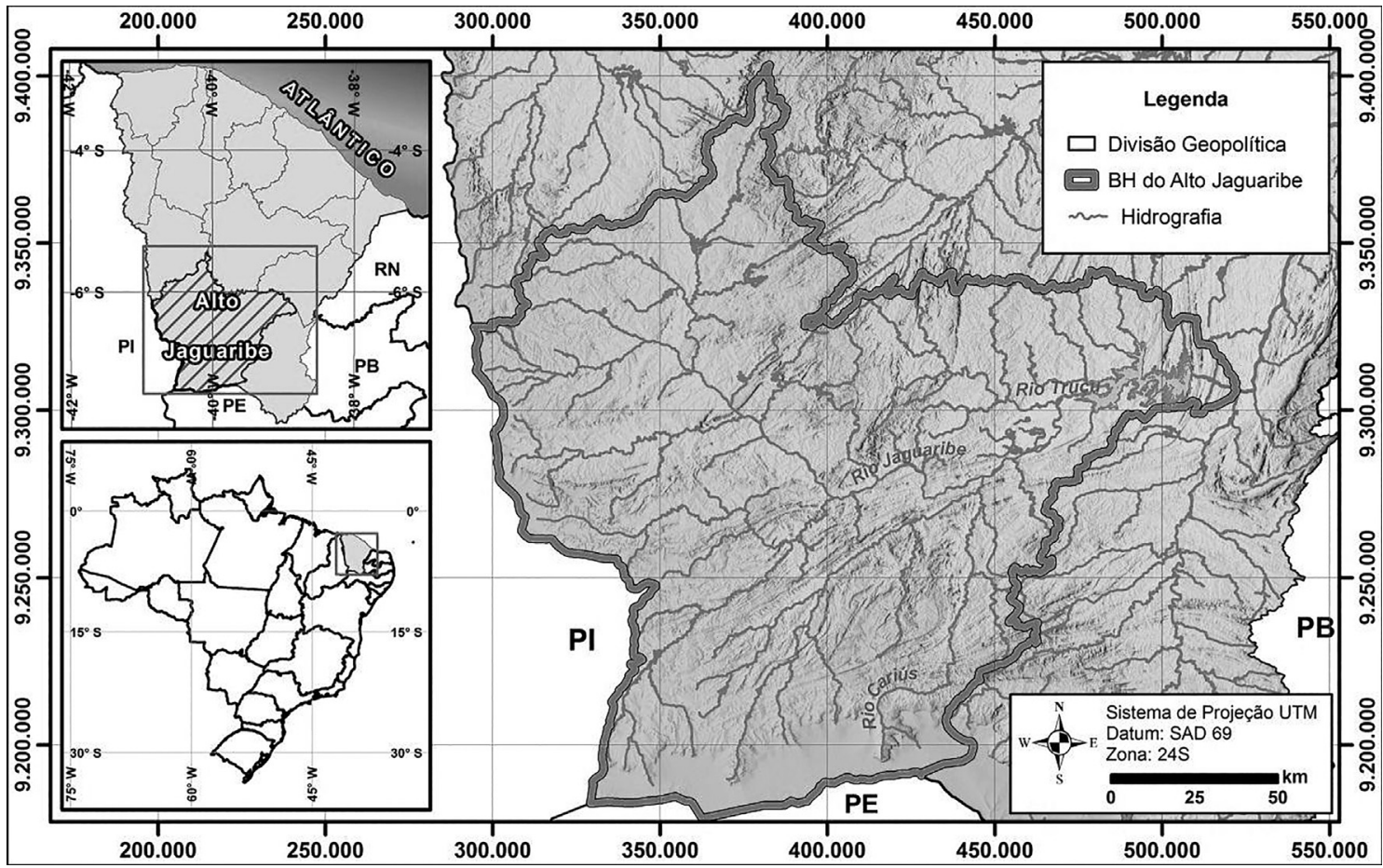

Figura 1 - Localização da área de estudo. 


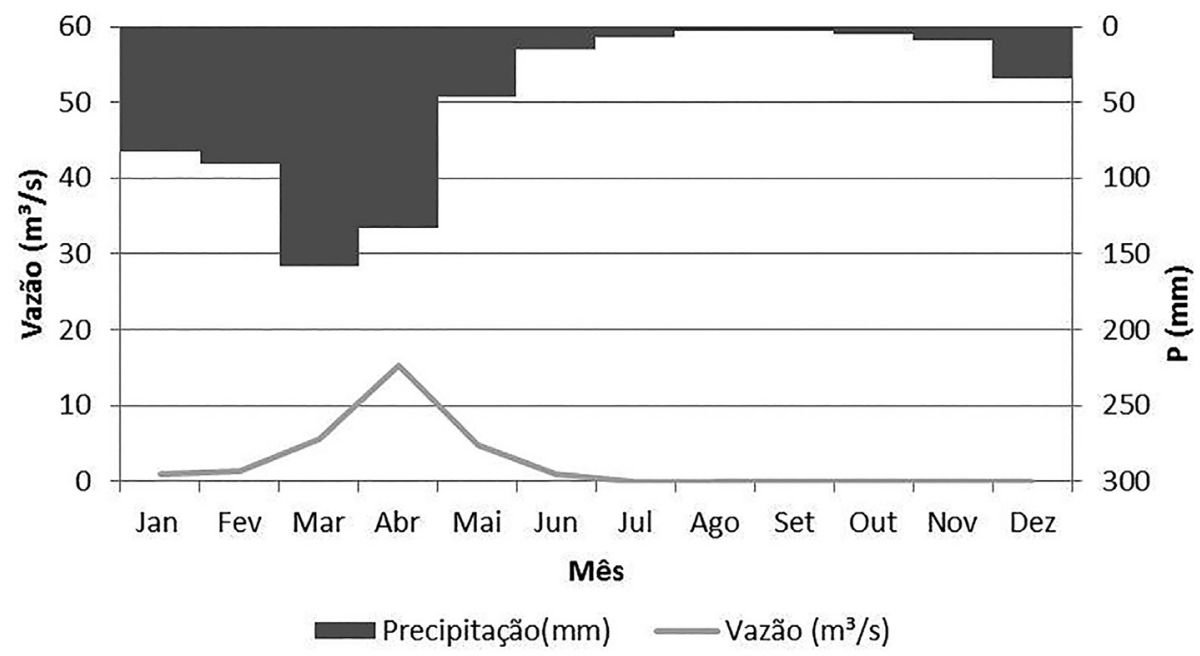

Figura 2 - Sazonalidade da precipitação na bacia e das vazões do rio Jaguaribe em Iguatu, na bacia hidrográfica do alto Jaguaribe.

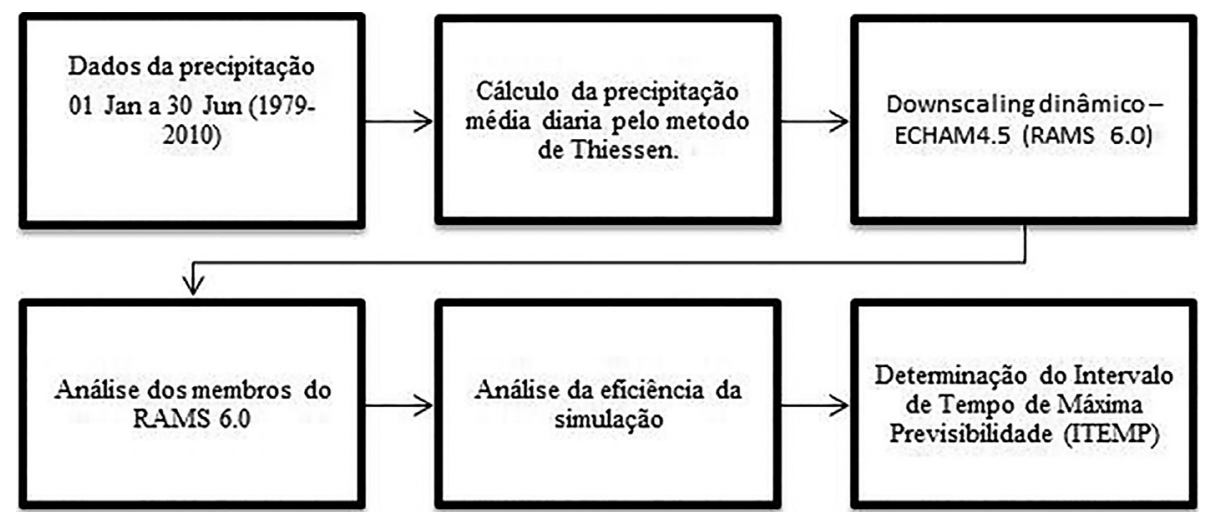

Figura 3 - Representação esquemática da ligação entre os modelos dinâmicos da atmosfera com a análise da efíciência dos modelos.

\subsection{Cálculo da precipitação média}

A precipitação observada diária foi integrada ao longo da bacia do alto Jaguaribe durante os primeiros seis meses do período 1979-2010. Para determinação da média espacial foi utilizado o método do polígono de Thiessen.

Este método atribui pesos relativos para cada posto, determinados pelas suas respectivas áreas de influência. As fronteiras dos polígonos são formadas pelas mediatrizes das linhas que unem dois postos adjacentes. Se há j postos, a área dentro da bacia designada para cada um é $A j$, e $P j$ é a precipitação registrada no $j$-ésimo posto, conforme a Eq. (1).

$$
\overline{P_{i}}=\frac{\sum P_{i, j} \cdot A_{i}}{A}
$$

onde $\overline{P_{i}}$ é a precipitação espacial média no dia $i, P_{i, j}$ representa a precipitação no dia $i$ no posto $j, A_{j}$ corresponde a área de influência do posto $j \mathrm{e} A$ é a área total da bacia.

$\mathrm{O}$ algoritmo aplicado considera, em cada dia $i$, somente os postos pluviométricos $j$ para os quais há dados observados.

\subsection{Modelagem dinâmica da precipitação}

O RAMS 6.0, está implementado na FUNCEME sendo usado para previsão de precipitação sobre o NEB desde $\mathrm{o}$ ano de 2001. Os dados oriundos do ECHAM4.5, geraram condições de fronteira de 10 membros para o modelo RAMS 6.0, os quais foram utilizados para comparar as precipitações do modelo com as precipitações observadas. Neste trabalho foram utilizados os dados do modelo regional RAMS, visto que Costa e Menezes Neto (2009) mostram que este possui desempenho superior para o Ceará. O RAMS 6.0 foi inicializado em modo de simulação com os dados do ECHAM4.5, sendo esta feita com um esquema de relaxamento Newtoniano (nudging), através de um termo adicionado as equações básicas, que faz com que o valor de cada variável nos diversos pontos de grade tenda àquele contido na informação de grande escala. A assimilação foi feita pelas fronteiras laterais, no topo e no centro do domínio do modelo (nudging lateral, topo e do centro), entretanto para os cálculos estatísticos esses pontos laterais foram desprezados, tendo em vista que os mesmos possuem ruídos causados pelo esquema do relaxamento newtoniano do modelo regional. 
O modelo RAMS 6.0 foi configurado com uma grade polar estereográfica, cobrindo todo o Nordeste Brasileiro e uma porção do Oceano Atlântico tropical circunvizinho, com 100 pontos em ambas as direções, com espaçamento horizontal de $30 \mathrm{~km}$ e centro localizado em $8^{\circ} \mathrm{S}, 40^{\circ} \mathrm{W}$. A grade vertical possui 38 níveis, apresenta espaçamento variável de $50 \mathrm{~m}$ nas proximidades da superfície a $1100 \mathrm{~m}$ próximo ao topo do modelo. As parametrizações adotadas incluem Mellor-Yamada (1974) para turbulência, Walko et al. (1995) para microfísica de nuvens, Chen-Cotton (1983) para radiação e Kuo modificada (1974) para convecção.

Embora geralmente não disponíveis, a umidade do solo e dados de temperatura, bem como outras propriedades do solo e as características da vegetação, são também necessária para a resolução espacial simulações do modelo. Nesta simulação optou por utilizar a umidade do solo inicial foi considerada a climatológica, apenas nos primeiros dias, e posteriormente as umidades estimadas pelo modelo. Os resultados desta simulação foram muito sensíveis à especificação das condições iniciais do campo de umidade do solo.

A Temperatura da Superfície do Mar (TSM) média mensal observada nos oceanos Pacífico, Atlântico e Índico nos meses de janeiro a dezembro (1979-2010) serviram de variáveis de contorno à superfície nas simulações do ECHAM4.5 e do RAMS 6.0. Os dados oriundos do ECHAM4.5, geraram a saída de 10 membros para o modelo RAMS 6.0, os quais foram utilizados para comparar as precipitações do modelo com as precipitações observadas.

O modelo regional foi rodado no período de 1979 a 2010, tendo como forçante de grande escala as condições de contorno do modelo global ECHAM4.5. As precipitações dos pontos de grade da região da bacia do Alto Jaguaribe foram usadas para a realização do experimento.

Nesse estudo foram utilizadas as simulações de precipitação do modelo RAMS 6.0 na bacia do alto Jaguaribe no primeiro semestre de cada ano, na escala mensal, cobrindo o período de 1979 a 2010. Aplicou-se uma grade de $30 \mathrm{~km} \mathrm{x}$ $30 \mathrm{~km}$, a partir da qual se obteve a precipitação média diária na bacia. A Fig. 4 apresenta a distribuição espacial dos postos utilizados e apenas os pontos de grade do RAMS 6.0 inserido na bacia.

Foi realizada ininterruptamente a rodada de simulação do alto Jaguaribe de 1979 a 2010, para 10 membros do ECHAM4.5, as forçantes de grande escala utilizadas nas simulações do RAMS 6.0 foram obtidas do conjunto das 10 integrações do ECHAM4.5.

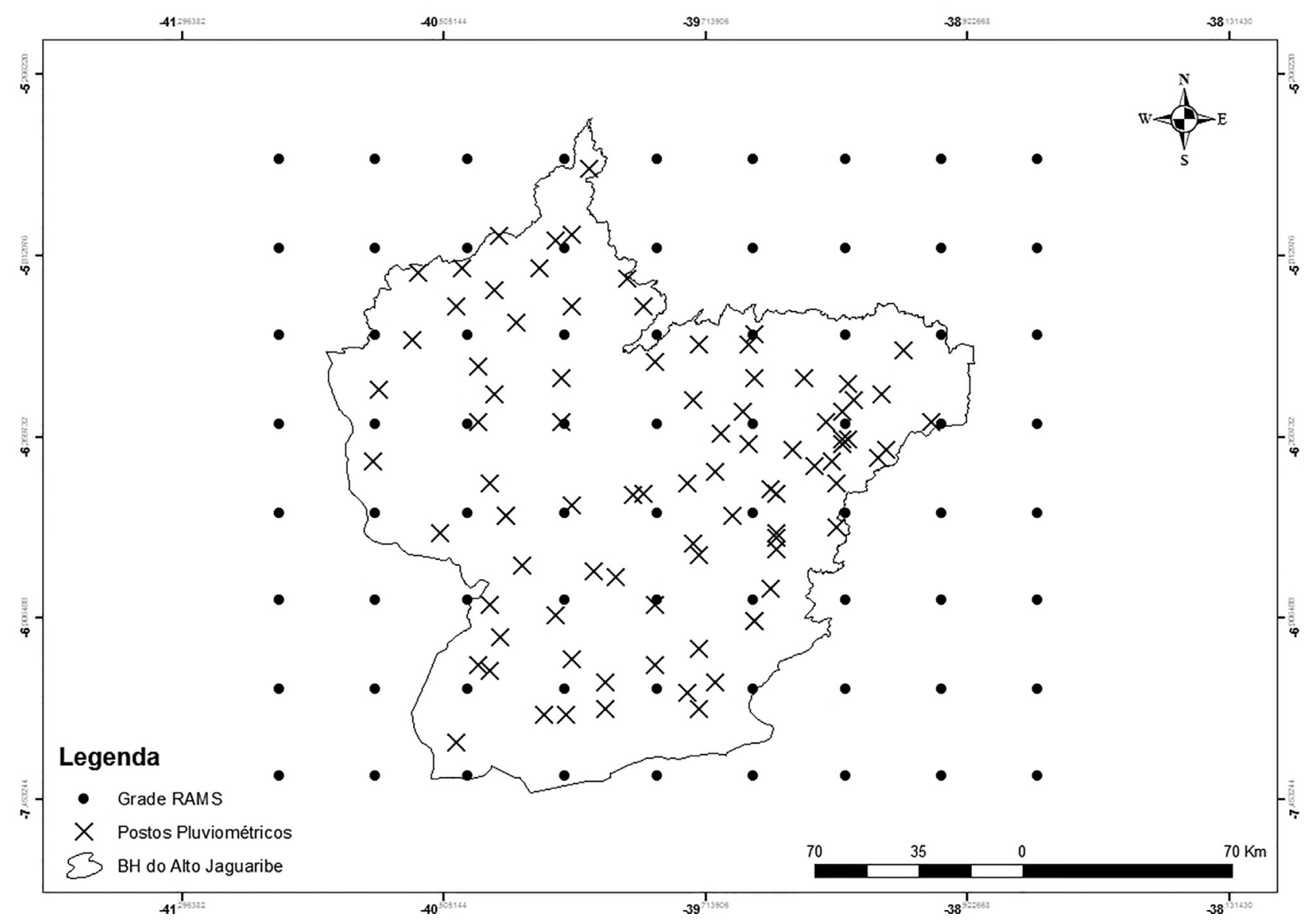

Figura 4 - Distribuição espacial dos postos pluviométricos e a grade do modelo RAMS 6.0 sobre a bacia do Alto Jaguaribe. 


\subsection{Análise dos membros do RAMS 6.0}

Vários autores (TOTH e KALNAY, 1993, MOLTENI et al., 1996, BRABO et al., 2007) corroboram que a melhor estimativa da atmosfera futura, especialmente para simulação de médio prazo, é o ensemble médio, principalmente numa aproximação determinística.

A previsão por ensemble consiste em calcular a média das previsões considerando-se que todos os membros sejam igualmente prováveis de ocorrerem, desta forma, não se atribui peso a nenhuma previsão específica. Neste estudo também se optou por usar o ensemble médio do modelo regional RAMS 6.0, para a bacia hidrográfica do Alto Jaguaribe.

\subsection{Análise da eficiência da simulação}

Diversos índices de analise de previsão são propostos e utilizados na literatura (WILKS, 2006; KULIGOWSKI, 2002; STEPHENSON, 2000; MESINGER, 2008) e poderiam também ser utilizados dentro da metodologia apresentada neste artigo. Independente do índice de desempenho utilizado ressalta-se a importância de entender sua formulação para poder avaliar mais coerentemente os resultados (HAMILL e JURAS, 2006).

Seguindo a proposta de Alves et al. (2012), optou-se por usar Heidke Skill Score (HSS) com o intuito de avaliar a eficiência do modelo RAMS 6.0 na área de estudo. O HSS é uma das estatísticas mais utilizadas para fornecer uma ideia da qualidade relativa de uma previsão. Geralmente, o sistema de referência é a própria climatologia obtida pela série histórica ou um sistema de previsão baseado na persistência (DOSWELL et al., 1990).

O HSS utiliza a proporção de acertos como métrica para calcular a qualidade relativa do sistema de previsão. Este índice possui valores entre - 1 e 1 que indicam, respectivamente, um modelo incapaz e um modelo perfeito de captar os fenômenos de precipitação. $\mathrm{O}$ valor zero para o
HSS significa que os valores das médias históricas são tão bons quanto o modelo de previsão. O HSS é calculado pela Eq. (2).

$$
H S S=\frac{\frac{1}{N} \sum_{i=1}^{k} N\left(F_{i} O_{i}\right)-\frac{1}{N^{2}}\left(\sum_{i=1}^{k} N\left(F_{i}\right) N\left(O_{i}\right)\right)}{1-\frac{1}{N^{2}} \sum_{i=1}^{k} N\left(F_{i}\right) N\left(O_{i}\right)}
$$

onde $\mathrm{N}(\mathrm{Fi}, \mathrm{Oi})$ denota o número de previsões na categoria $\mathrm{i}$ que tem observações na categoria i, N (Fi) indica o número total de previsões na categoria $\mathrm{i}, \mathrm{N}(\mathrm{Oi})$ significa que o número total de observações na categoria i e $\mathrm{N}$ o número total de observações, $\mathrm{k}$ é o número de classes.

\subsection{Determinação do Intervalo de Tempo de Máxima Previsibilidade (ITEMP)}

Como critério de avaliação do ITEMP da precipitação na bacia hidrográfica do Alto Jaguaribe, foram considerados os valores do HSS, usados para comparar a precipitação média observada na bacia e a precipitação media simulada na bacia pelo modelo RAMS 6.0, sendo o maior valor de HSS igual a melhor ITEMP.

Os processos iniciais da simulação foram feitas com a simulação variando no tempo em conjunto de $1,2,10,15$, 21, 30, 45, 60, 120 e 180 dias, sendo analisados os HSS para cada intervalo de tempo da simulação.

A Fig. 5 mostra o croqui do algoritmo desenvolvido para a determinação do ITEMP na bacia hidrográfica do Alto Jaguaribe, com as etapas dos cálculos e as condições consideradas nesse trabalho.

A busca pelo ITEMP se dá pelo fato principalmente da necessidade das ações de gerenciamento do sistema hídrico, serem tomadas no começo do primeiro semestre, no planejamento da quadra chuvosa no Ceará, principalmente para mitigar eventos extremos, como as secas e as cheias, que tanto afetam a região do estudo.

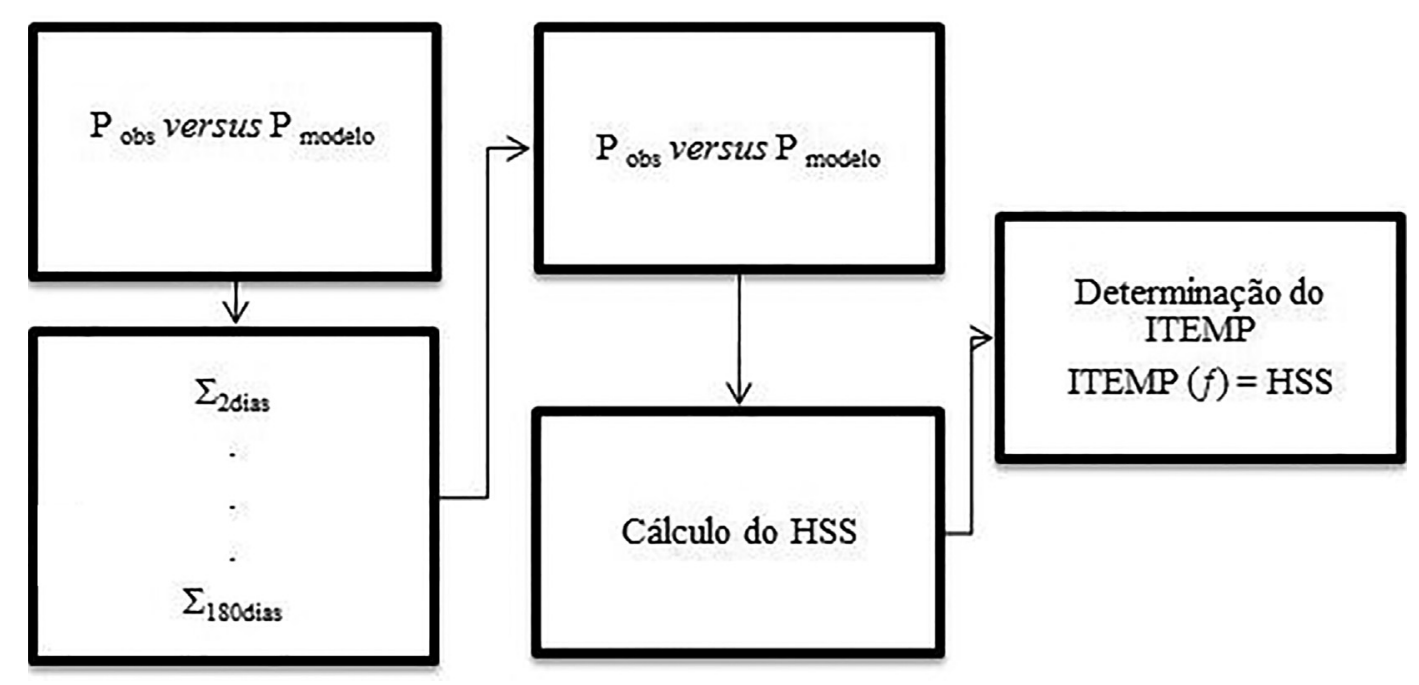

Figura 5 - Algoritmo metodológico para determinação do ITEMP. 


\section{Resultados e Discussões}

\subsection{Análise da precipitação}

A Fig. 6 mostra as comparações entre precipitações mensais observadas e modeladas pelo modelo RAMS 6.0. Percebe-se que em quase todo período analisado o modelo subestimou os dados observados de precipitação média na bacia, mostrando deficiência na estimativa principalmente de precipitações maiores de $100 \mathrm{~mm} / \mathrm{mês}$. Esse erro pode esta relacionada a fatores dos dados observados na bacia assim como as condições iniciais da modelagem atmosférica.

Alguns dos resultados obtidos podem ser explicados por Pielke (2012) e Castro et al. (2005), que levantaram algumas limitações referentes ao downcaling dinâmico, como: considerar nas condições iniciais todas as forçantes do modelo; deficiência em previsões multidecadais; o refinamento da regionalização; o domínio dos modelos regionais e por fim as condições de contorno laterais.

Outro fator que pode influenciar nos resultados é a capacidade dos modelos atmosféricos em detectar os sistemas meteorológicos atuantes na área de estudo, como por exemplo, as influências de frente frias na pré-estação chuvosa na região, e tendo seu desempenho satisfatório durante o período da aproximação da ZCIT, por exemplo. No primeiro trimestre nota-se maior discrepância sobre, a bacia hidrográfica presumindo que o modelo RAMS 6.0 tenha menos destreza na previsão climática sobre a bacia nesse período, possivelmente devido às incertezas e a variabilidade dos sistemas meteorológicos na bacia. Já durante fevereiro, nota-se um maior ordenamento na precipitação sobre a bacia.

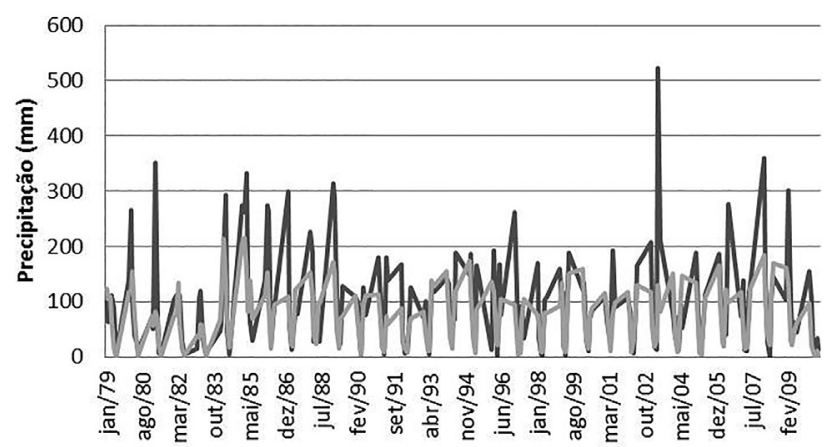

$$
\text { - Obs Modelo }
$$

Figura 6 - Análise das precipitações médias mensais observadas vs. modelada no período 1979-2010.
Ainda no escopo da análise integrada no espaço, é interessante questionar o desempenho das estimativas de ocorrência de chuva em função da intensidade da chuva observada.

O primeiro trimestre JAN-FEV-MAR apresenta maior parte dos dados subestimando as simulações de precipitação ao longo do tempo, entretanto os valores dos vieses mostram uma pequena variabilidade em todo período.

Lima e Alves (2009) aplicaram o modelo RAMS 6.0 na bacia do rio São Francisco e verificaram subestimativas das médias mensais, e erros sobre a faixa da região do estudo, o que segundo os autores pode estar associado às parametrizações de convecção e efeitos de topografia.

No fim da estação chuvosa na bacia, o modelo comporta-se com uma melhor eficiência, principalmente nos meses de maio e junho, com pequenas variações comparando as precipitações observadas e modeladas sobre a bacia.

\subsection{Análise da eficiência}

Para uma melhor habilidade de previsão deve-se aumentar o numero de membros dos conjuntos do modelo atmosféricos. A Tabela 1 mostra a variação dos valores do HSS nas escalas de 1, 2, 10, 15, 21, 30, 45, 60, 120 e 180 dias, com o objetivo de encontrar o melhor valor de HSS na avaliação sazonal da precipitação, ou seja, encontrar o ITEMP.

Os valores do HSS, apresentados na Tabela 1, onde valores de HSS a partir do decimo dia apresentam skill > 0,35; ou seja, o modelo apresenta uma razoável destreza nesse intervalo de tempo. A partir do dia 15 o modelo apresenta valores de HSS bastante satisfatórios em escala de bacias hidrográficas, com HSS $>0,37$. Nota-se, que a partir do intervalo de 30 dias, com HSS $=0,54$, e de 45 dias, com HSS $=0,62$, o modelo tende a melhora habilidade de previsão.

A habilidade típica das previsões sazonais utilizando o HSS que é obtido operacionalmente com base em modelos climáticos globais sobre a terra é da ordem de 0,10 (Mason et al., 1999).

$\mathrm{O}$ valor ótimo procurado encontra-se no intervalo de 45 dias, com HSS igual a 0,62 ; o que mostra que o modelo nesse intervalo apresenta melhor habilidade em prever as precipitações na bacia do Alto Jaguaribe.

Lima e Alves (2009) testaram vários parâmetros estatísticos na avaliação de desempenho da precipitação em bacias hidrográficas, com e sem correções PDFs, usando a

Tabela 1 - Variação dos HSS, para a bacia do Alto Jaguaribe.

\begin{tabular}{lcccccccc}
\hline Tempo (dias) & 1 & 2 & 10 & 15 & 21 & 30 & 45 & 60 \\
\hline HSS (ECHAM 4.5) & 0.189 & 0.198 & 0.222 & 0.298 & 0.321 & 0.435 & 0.512 & 0.386 \\
HSS (RAMS 6.0) & 0.212 & 0.238 & 0.338 & 0.376 & 0.434 & 0.547 & 0.622 & 0.479 \\
\hline
\end{tabular}


grade de um modelo regional, para um período de 30 anos. Quanto à correlação, os autores não encontraram diferenças muito significativas no coeficiente de correlação e na razão entre os desvios padrões da precipitação prevista e observada, embora os valores sejam um pouco maiores em magnitude com os dados de precipitação corrigidos.

Paz e Collischonn (2011) testaram vários indices de desempenho de previsão de precipitações na bacia hidrografica do Rio Grande, e verificaram que a análise das séries temporais dos índices de desempenho possibilitaram avaliar a existência de sazonalidade na destreza em estimar ocorrência/não ocorrência de chuva. Além disso, eles retrataram a variação espacial do desempenho e evidenciaram tendências espaciais em superestimar/subestimar a ocorrência de precipitação em porções distintas da bacia.

A Fig. 7 mostra a variação os HSS ao logo do intervalo de tempo, apresentando que os melhores valores de HSS encontram-se no intervalo do vigesimo primeiro dia ao secsagesimo dia, e os valores de HSS com menor habilidade de previsão encontram-se nos primeiros dias da previsão.

Embora as análises apresentadas possuam limitações, já que utilizaram apenas uma realização de cada um dos modelos, nota-se que a regionalização com RAMS 6.0 adiciona informações às previsões do ECHAM 4.5, corrigindo alguns erros sistemáticos deste. Futuramente, pretende-se analisar um maior número de previsões regionais, ou utilizando várias previsões do ECHAM 4.5, ou perturbando a TSM persistida e realizando várias previsões como o RAMS 6.0 partindo de uma previsão do ECHAM 4.5, ou seja, construindo um conjunto de previsões trimestrais e analisando os resultados probabilisticamente.

\section{Conclusões}

O Intervalo de Tempo de máxima previsibilidade (ITEMP) dá-se para simulações com intervalos superiores há 45 dias (15 de Fevereiro) com HSS acima de 0,62, podendo ser usada essa simulação para auxiliar o geren-

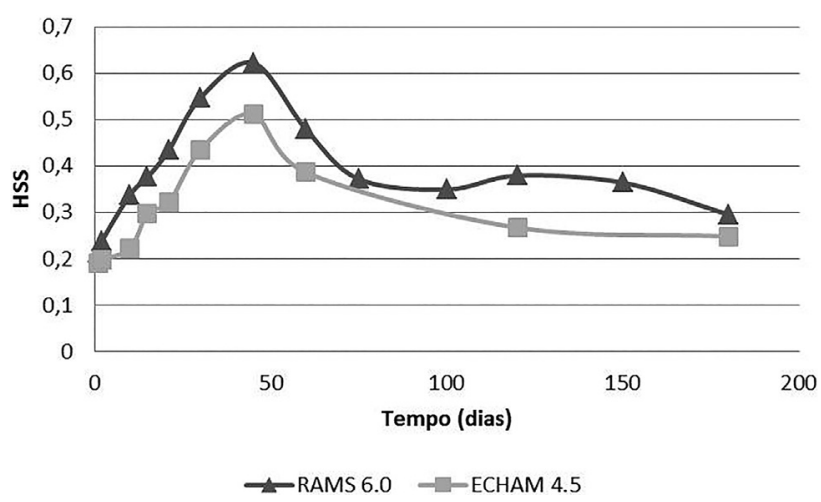

Figura 7 - Valores de HSS para a simulação da precipitação na bacia do Alto Jaguaribe. ciamento dos recursos hídricos na bacia hidrográfica do Alto Jaguaribe, no Semiárido brasileiro.

O modelo apresenta deficiência no intervalo de tempo de até 10 dias, mostrando ser miope para previsão climatica em um pequeno intervalo de tempo, provavelmente devido as condições inicias de umidade do solo e uma destreza superior, com HSS em média maiores que 0,44 para um horizonte maior de tempo, podendo está associada a previsão do modelo em detectar sistemas meteorológicos de grande escala.

A metodologia utilizada nesse trabalho identifica o ITEMP, podendo assim auxiliar ao apoio e ao desenvolvimento de ferramentas no sistema de previsão hidrometeorológicas, sendo predecessor para o objetivo futuro, de um acoplamento eficiente do conjunto precipitação-escoamento na bacia do Alto Jaguaribe.

Vale a pena ressaltar que nos resultados apesar da simulação ser climática há um máximo de previsão na escala de 45 dias (intrassazonal), que sugere o uso da previsão climática em simulação/previsão abaixo da sazonalidade, podendo ter potencial em acoplamento em simulações ou previsões de vazões para uso em reservatórios hídricos.

\section{Agradecimentos}

Os autores agradecem aos Editores e Revisores da RBMET pelas críticas e sugestões que melhoraram a apresentação e o conteúdo científico do artigo e a FUNCEME pela disponibilidade dos dados.

\section{Referências}

ALVES, J.M.B.; CAMPOS, J.N.B.; SOUZA FILHO, F.A.; MUNCUNILL, D.F.; FERREIRA, A.G.; Sun, L.; Moura, A.D. Uma avaliação preliminar de simulações climáticas de um modelo regional espectral aninhado em um modelo global (ECHAM4.5) sobre o setor norte da região Nordeste do Brasil (1971-2000). Revista Brasileira de Meteorologia, v. 20, n. 2, p. 191-206, 2005.

ALVES, J.M.B.; CAMPOS, J.N.B.; MARTINS, E.S.P.R.; Muncunill, D.F.; SOMBRA, S.S.; Souza Filho, F.A. Um estudo inter-comparativo de previsão sazonal estatística-dinâmica de precipitação para o Nordeste do Brasil. Revista Brasileira de Meteorologia, v. 24, p. 20-29, 2007.

ALVES, J.M.B.; CAMPOS, J.N.B.; NASCIMENTO, L.S.V. Sensibilidade intrasazonal de um downscaling dinâmico de precipitação (1971-2000): uma análise na bacia hidrográfica do açude Castanhão-Ce. Revista Brasileira de Meteorologia, v. 23, p. 73-87, 2008.

ALVES, J.M.B.; CAMPOS, J.N.B.; SERVAIN, R. Reservoir management using coupled atmospheric and hydrological models: The brazilian semi-arid case. Water resources management, v. 26, n. 5, p. 1365-1385, 2012. 
CASTRO, C.L.; R.A. PIELKE SR.; LEONCINI,G. Dynamical downscaling: Assessment of value retained and added using the Regional Atmospheric Modeling System (RAMS). Journal of Geophysical Research, v. 110, p. 1-21, 2005.

CHEN, C.; COTTON, W. R. A one-dimensional simulation of the stratocumulus-capped mixed layer. BoundLayer Meteor, v. 25, p. 289-321, 1983.

COSTA, A.A.; MENEZES NETO, O.L. Modelagem de mesoescala da variabilidade climática interanual sobre o nordeste brasileiro. Anais do III Simpósio Internacional de Climatologia, 2009.

DOSWELL, C.A.; JONES, R.D.; KELLER, D.L. On summary measures of skill in rare forecasting based on contingency tables. Weather and Forecasting, v. 5, p. 576-585, 1990.

FUNCEME. Fundação Cearense de Meteorologia e Recursos Hídricos do Ceará. www.funceme.br, acesso em 10 de janeiro de 2013.

GODDARD, L.; MASON, J.S.; ZEBIAK, S.E.; ROPELEWSKI, C.R.; BASCHER, R.; CANE, M.A. Current approaches to seasonal-to-interanual climate predictions. International Journal of Climatology, Londres, v. 21, p. 1111-1152, 2001.

HAMILL, T.; JURAS, M. Measuring forecast skill: is it real skill or is it the varying climatology? Quarterly Journal of the Royal Meteorological Society, v. 132, p. 2905-2923, 2006.

JASPER,K.; KAUFMANN, P. Coupled runoff simulations as validation tools for atmospheric models at the regional scale. Quarterly Journal of the Royal Meteorological Society, v. 129, p. 673-693, 2003.

KUO, H. L. Further studies of the parameterization of the influence of cumulus convection on large-scale flow. Journal of the Atmospheric Sciences, v. 31, n. 5, p. $1232-1240,1974$.

LIMA. J.P.R.; ALVES. J.M.B. Um estudo de downscaling dinâmico de precipitação intrasazonal acoplado a modelo chuva-vazão na bacia hidrográfica altomédio São Francisco. Revista Brasileira de Meteorologia, v.24, n.3, 323-338, 2009.

KULIGOWSKI, R.A. Self-calibrating real-time GOES rainfall algorithm for short-term rainfall estimates. Journal of Hydrometeorology, v. 3, p. 112-130, 2002.

MASON, S.J.; GODDARD, L.; GRAHAM, N.E.; YULAEVA, E.; SUN, L.; ARKIN, P.A. The IRI seasonal climate prediction system and the 1997/98 El Niño event. Bulletin of the American Meteorological Society, v. 80, p. 1853-1973, 1999.

MELLOR, G.; YAMADA, T. A hierarchy of turbulence closure models for atmospheric boundary layers. Journal of the Atmospheric Sciences, v. 31, p. 17911806, 1974.
MESINGER, F. Bias Adjusted Precipitation Threat Scores. Advances in Geosciences, v.16, p. 137-142, 2008.

MILLER, N.L.; KIM, J.; HARTMAN, R.K.; FARRARA, J. Downscaling climate on stream flow study on Southwestern on Unite States. Journal American Water Resources Association, v. 35, p. 1525-1537, 1999.

MOLTENI, F.; BUIZZA, R.; PALMER, T.N.; PETROLIAGIS, T. The ECMWF ensemble prediction system: Methodology and validation. Quarterly Journal of the Royal Meteorological Society, v. 122, p. 73-119, 1996.

NOBRE, P.; MOURA, A. D.; SUN,L. Dynamical downscaling of seasonal climate prediction over Nordeste Brazil with ECHAM3 and NCEP's regional spectral models at IRI. Bulletin of the American Meteorological Society, v. 82, p. 2787-2796, 2001.

OLIVEIRA, K. F. Previsão de vazão em uma bacia do semiárido usando previsões climáticas numéricas de precipitação. 2006. 77 p. Dissertação de Mestrado em Engenharia Civil e Ambiental. Universidade Federal de Campina Grande. Departamento de Engenharia Civil e Ambiental. Campina Grande-PB. 2006.

PAZ, A. R. D.; COLLISCHONN, W. Avaliaçãode estimativas de campos de precipitação para modelagem hidrológica distribuída. Revista Brasileira de Meteorologia, v.26, n. 1, p. 109-120, 2011.

PIELKE, R. A.; COTTON, W. R.; WALKO, R. L. A comprehensive meteorological modeling system-RAMS. Meteorology and Atmospheric Physics, v. 49, n. 1, p. 69-91, 1992.

STEPHENSON, D.B. Use of the "odds ratio" for diagnosing forecast skill. Weather and Forecasting, v. 15, p. 221-232, 2000.

SUN, L.; MONCUNILL, D.V.; Li, H.; MOURA, A.D.; SOUZA FILHO, F.A. Climate downscaling over Nordeste, Brazil, using the NCEP RSM97, Journal of Climate, v. 18, p. 551-567, 2005.

TOTH, Z.; KALNAY, E. Ensemble forecasting at NMC: The generation of perturbations. Bulletin of the American Meteorological Society, v. 74, p. 2317-2330, 1993.

WALKO, R.L.; COTTON, W.R.; MEYERS, M.P.; HARRINGTON, J.Y. New RAMS cloud microphysics parameterization Part I: the single-moment scheme. Atmospheric Research, v. 38, n. 1, p. 29-62, 1995.

WEAVER, C.P.; ROY, S.B.; AVISSAR, R. Sensitivity of simulated mesoscale atmospheric circulations resulting from landscape heterogeneity to aspects of model configuration. Journal of Geophysical Research, v. 107, p. 1-21, 2002.

WILKS, D.S. Statistical methods in the atmospheric sciences. 2. ed. San Diego: Academic Press, v. 91, 2006.

All the contents of this journal, except where otherwise noted, is licensed under a Creative Commons Attribution License CC-BY. 